Ashdin Publishing

Journal of Generalized Lie Theory and Applications

Vol. 4 (2010), Article ID G090902, 11 pages

doi:10.4303/jglta/G090902

\title{
On the product of conjugacy classes in unitary group and singular connections
}

\author{
Jafar SHAFFAF \\ Department of Mathematical Sciences, Shahid Beheshti University, G.C., \\ P.O. Box 1983963113, Tehran, Iran \\ E-mail: j_shaffaf@sbu.ac.ir
}

\begin{abstract}
We are attempting to give a new proof to the problem of characterization of the support of the product of conjugacy classes in the compact Lie group $\mathrm{SU}(n)$ without any reference to the Mehta-Seshadri theorem in algebraic geometry as it was the case in [1].
\end{abstract}

2000 MSC: 20E45, 22E10, 14H60, 55R50

\section{Introduction}

It is well known that the product of two conjugacy classes in $\mathrm{SU}(n)$ can be described by a set of linear inequalities on the Lie algebra of its maximal torus [1], and that these inequalities are a re-statement of the property of (semi)-stability of certain vector bundles on $\mathbb{C} P(1)$ with three (or more) points removed. The proof in [1] depends on a theorem of Mehta-Seshadri [9] or equivalently can be reformulated in terms of gauge theory of singular flat connections. For a survey on the case of sum of Hermitian matrices, see the descriptive papers [6, 7]. The purpose of this paper is to give a direct and simple proof of the description of the product of two conjugacy classes in $\mathrm{SU}(n)$ which makes no use of the theorem of Mehta-Seshadri or gauge theory. The main technical tools are an analogue of the Gauss-Bonnet theorem generally known as the Gauss-Chern formula (see [4]) and a well-known decomposition of the curvature tensor [8]. These methods are quite elementary and in the course of the proof we give a clear exposition of some of ideas related to vector bundles on marked Riemann surfaces.

The main result about the product of two conjugacy classes in $\mathrm{SU}(n)$ is given by the following theorem.

Theorem 1.1. Let $\alpha=\left(\alpha_{1} \geq \alpha_{2} \geq \cdots \alpha_{n}\right), \beta$, and $\gamma$ be $n$-tuples of real numbers in $(-1,1]$ such that $\sum \alpha_{j}=\sum \beta_{j}=\sum \gamma_{j}=0$. Let $C_{\alpha}$ denote the conjugacy class in $\mathrm{SU}(n)$ determined by the eigenvalues $e^{\pi i \alpha_{j}}$. Then a conjugacy class $C_{\gamma}$ occurs in the product $C_{\alpha} C_{\beta}$ if and only if

$$
S(\alpha, \beta, \gamma)+\sum_{i \in I} \alpha_{i}+\sum_{j \in J} \beta_{j}+\sum_{k \in K} \gamma_{k} \leq 0
$$

where $S(\alpha, \beta, \gamma)$ is an integer and $I, J, K \subset\{1,2, \ldots, n\}$ are subsets of the same cardinality $l \leq n$. The exact description of the integer $S(\alpha, \beta, \gamma)$ and the subsets $I, J$, and $K$ is given in Section 4 below. 
In Section 2 we give generalities about vector bundles on marked Riemann surfaces and their relationship with products of conjugacy classes. Although the proof of the main theorem makes no use of algebraic geometry of parabolic vector bundles (which was used in $[1,9]$ ), we give precise definitions which may be useful in giving a direct and simple algebraic proof of the Mehta-Seshadri theorem which was also proven by Biquard [4] using differential geometric methods. In Section 3 we show the (semi)-stability of vector bundles constructed in Section 2 from conjugacy classes by using the Gauss-Chern formula and a decomposition of the curvature tensor of sub-bundles. Finally in Section 4 we relate the geometric concepts of Section 3 to our main problem and give an explicit description of the integer $S(\alpha, \beta, \gamma)$ and the subsets $I, J$, and $K$ thereby proving the main theorem. This description involves quantum multiplication of Schubert cycles.

\section{Generalities on vector bundles on marked Riemann surfaces}

Let $M^{*}$ be a compact Riemann surface with $m$ marked points or cusps $p_{s}, s=1, \ldots, m$. Throughout the paper the subscript $s$ refers to the cusps. Set $M=M^{*} \backslash\left\{p_{1} \cdots p_{m}\right\}$. We assume that the Euler characteristic of $M$ is negative so that its universal covering space is the upper half-plane $\mathcal{H}$. In addition, we assume that $m \geq 1$ so that the fundamental group of $M$ is the free group $\mathrm{F}_{2 g+m-1}$ on $2 g+m-1$ generators. The fundamental group of a neighborhood of a cusp $p_{s}$ is isomorphic to $\mathbb{Z}$. A neighborhood $U_{s}$ of $p_{s}$ is uniformized by the subset $\mathcal{H}_{T}=\{z \in \mathcal{H} \mid \Im(z)>T\}$ for some large $T>0$ and the action of the local fundamental group is by translation $z \stackrel{k}{\longrightarrow} z+k$. Let $\rho: \pi_{1}(M, \zeta) \rightarrow \mathrm{SU}(n)$ and note that for $\gamma \in \pi_{1}(M, \zeta) \simeq \mathrm{F}_{2 g+m-1}, \rho(\gamma)$ is conjugate in $\mathrm{U}(n)$ to a matrix of the form

$$
\left(\begin{array}{ccc}
e^{2 \pi i \gamma_{1}} & & O \\
& \ddots & \\
O & & e^{2 \pi i \gamma_{n}}
\end{array}\right) .
$$

Let $E=E_{\gamma}$ denote the diagonal matrix with entries $\left[\gamma_{1}, \ldots, \gamma_{n}\right]$ and through the paper we assume that all the eigenvalues are integer numbers in $[-1,1)$. The given representation $\rho$ gives a holomorphic vector bundle $E_{\rho}$ of rank $n$ on $M$ as a fibre product in the usual manner. For each $j$ let $\mathcal{F}_{T}=\left\{z \in \mathcal{H}_{T} \mid-\frac{1}{2}<\Re(z) \leq \frac{1}{2}\right\}$. For $z \in \mathcal{H}_{T}$ define $l \in \mathbb{Z}$ to be the unique integer $l$ such that $z-l=z_{1} \in \mathcal{F}_{T}$. Set

$$
g: \mathcal{H}_{T} \longrightarrow \mathrm{GL}(n, \mathbb{C}), \quad g(z)=z_{1}^{N E \gamma_{j}} \rho\left(\gamma_{j}^{-l}\right),
$$

in which $N$ is the least integer number such that $N \gamma_{j} \in \mathbb{Z}$ for $1 \leq j \leq n$ (the choice of $N$ is for the matter of holomorphicity of the map $g$ ). Note that

$$
g(z+1)=z_{1}^{N E \gamma_{j}} \rho\left(\gamma_{j}^{-l-1}\right)=g(z) \rho(-1) .
$$

Since a holomorphic vector bundle on $M$ is holomorphically trivial, it can be extended to any other vector bundle of rank $n$ on $M^{\star}$. We will describe a specific extension of $E_{\rho}$ to $M^{\star}$ and throughout we will only consider this extension. Identifying $U_{j}$ with the unit disc with the origin removed $D^{\star}$, we glue $E_{\rho \mid U_{j}} \simeq U_{j} \times \mathbb{C}^{n}$ with $D^{\star} \times \mathbb{C}^{n}$ by the map

$$
U_{j} \times \mathbb{C}^{n} \longrightarrow D^{\star} \times \mathbb{C}^{n}, \quad(z, \xi) \longrightarrow\left(z_{1}, g(z) \xi\right) .
$$

This gives us the extension of $E_{\rho}$ to $M^{\star}$ which we call the standard extension and denote by $E_{\rho}^{\star}$ if necessary for emphasis. In view of the standard extension we define the (first) Chern class of $E_{\rho}$ as $c_{1}\left(E_{\rho}^{\star}\right) \in H^{2}\left(M^{\star} ; \mathbb{Z}\right) \simeq \mathbb{Z}$. 
Lemma 2.1. The line bundle $\operatorname{det} E_{\rho}^{*}$ is holomorphically trivial and consequently the Chern class of $E_{\rho}^{*}$ is zero.

Proof. By the above construction the transition functions of $E_{\rho}^{\star}$ are given by

$$
g_{\alpha \beta}(z)=z^{\alpha} P z^{-\beta} P^{-1},
$$

where matrices $E$ and $E^{\prime}$ are diagonal matrices in $\mathrm{U}(n)$ with diagonal entries $\left[\alpha_{1}, \ldots, \alpha_{n}\right]$ and $\left[\beta_{1}, \ldots, \beta_{n}\right]$, and $P \in \mathrm{U}(n)$.

Precisely, according to the construction of the trivialization around the point 0 and 1 in the previous step, for each trivialization we consider the diagonalization of the generator of the stabilizer subgroup of the corresponding cusp, so in general we cannot simultaneously diagonalize the two matrix corresponding to the parabolic points 0 and 1 . Let

$$
E=\alpha=\left(\begin{array}{ccc}
\alpha_{1} & & O \\
& \ddots & \\
O & & \alpha_{n}
\end{array}\right)
$$

and the matrix $E^{\prime}$ is conjugate to the diagonal matrix $\beta$ by a matrix $P \in \mathrm{SU}(n)$ as follows:

$$
P^{-1} E^{\prime} P=\beta=\left(\begin{array}{ccc}
\beta_{1} & & O \\
& \ddots & \\
O & & \beta_{n}
\end{array}\right) .
$$

So by the construction for the transition function $g_{\alpha \beta}: U_{\alpha} \cap U_{\beta} \rightarrow \operatorname{GL}(n, \mathbb{C})$ we have

$$
g_{\alpha \beta}(z)=z^{\alpha} P z^{-\beta} P^{-1} .
$$

The Chern class of the bundle $E_{\rho}^{*}$ is equal to the Chern class of the determinant bundle, which is a line bundle with transition function

$$
\varphi_{\alpha \beta}(z)=\operatorname{det} g_{\alpha \beta}(z)=\frac{z^{\sum_{i=1}^{n} \alpha_{i}}}{z^{\sum_{i=1}^{n} \beta_{i}}}
$$

proving that $\operatorname{det} E_{\rho}^{*}$ is in fact a trivial bundle and hence has vanishing Chern class.

To define the notion of parabolic bundle, at each cusp $p_{j}$ we fix a flag $E_{1}^{p} \subset E_{2}^{p} \subset \cdots \subset E^{n}$ subject to the requirement that the subspace $E_{k}^{p}$ is invariant under the action of $\rho\left(\gamma_{j}\right)$ which acts as the scalar $e^{2 \pi i \alpha_{k}}$ on $E_{k} / E_{k-1}$. Let $\mathcal{E} \subset E_{\rho}^{\star}$ be a holomorphic sub-bundle with fibre dimension $r$. Define the integers $a_{1}<a_{2}<\cdots<a_{r}$ as follows: $a_{1}$ is the smallest integer such that $\operatorname{dim}\left(E_{p_{j}} \cap E_{a_{1}}\right)=1$; and $a_{l}$ is the smallest integer such that $\operatorname{dim}\left(E_{p_{j}} \cap E_{a_{l}}\right)>$ $\operatorname{dim}\left(E_{p_{j}} \cap E_{a_{l-1}}\right)$. Define the parabolic degree of $\mathcal{E}$ as

$$
\operatorname{pardeg}(\mathcal{E})=c(\mathcal{E})+\sum_{s} \sum_{k=1}^{l} \alpha_{s, a_{k}} .
$$

The parabolic slope $\mu(E)$ is

$$
\mu(E)=\frac{\operatorname{pardeg}(E)}{\operatorname{rk}(E)} .
$$


Given a holomorphic sub-bundle $F \subseteq E$ of rank $r$, one obtains a parabolic structure on $F$ as follows. An ascending flag in the fiber $F_{p}$ at marked (cusp) point $p$ is obtained by removing from

$$
F_{p} \cap E_{p, 1} \subseteq F_{p} \cap E_{p, 2} \subseteq \cdots \subseteq F_{p} \cap E_{p, n}=F_{p}
$$

those terms for which the inclusion is not strict (note that since the vector space $F_{p}$ is an $r$-dimensional vector space, exactly $r$ inclusions of the above sequence of inclusions are strict). The parabolic weights for $F$ are $\beta_{j}=\alpha_{k_{j}}$, where $k_{j}$ is the minimal index such that $F_{p, j} \subseteq E_{p, k_{j}}$ where $F_{p, j}=F_{p} \cap E_{p, j}$.

A parabolic sub-bundle of $E$ is a holomorphic sub-bundle $F \subset E$ whose parabolic structure is the one induced from the inclusion. We say the parabolic bundle $E \rightarrow X$ is parabolic semistable (stable) if $\mu(F) \leq \mu(E)(\mu(F) \supsetneqq \mu(E))$ for all parabolic sub-bundles $F \subset E$. Now we introduce the necessary tools for dealing with parabolic vector bundle from the differential geometric viewpoint.

\section{Singular Gauss-Chern formula}

In this section we relate the weights defined in the previous section for parabolic bundle more intrinsically in a way that these numbers is corresponded to the geometry of the bundle. Assume that the bundle $E$ over the Riemann surface $X$ is parabolic at the cusp point $p$ and equipped with a hermitian metric $h$ smooth on $X-\{p\}$ and degenerate at $p$ and in some sense which will be made precise later this metric is adaptive with the parabolic structure.

Let $\mathcal{C}$ be the space of holomorphic structures over $E$ or more precisely the space of operators

$$
\bar{\partial}^{E}: C^{\infty}(E) \longrightarrow C^{\infty}\left(\Omega^{0,1} \otimes E\right), \quad \bar{\partial}^{E}(f s)=f \bar{\partial}^{E}(s)+(\bar{\partial} f) s .
$$

Let $A$ be the space of $h$-unitary connections which the associated holomorphic structure $\bar{\partial}^{E} \in \mathcal{C}$. In other words, this is the space of $h$-unitary connections which is smooth on $X-\{p\}$ whose $(0,1)$ part is smooth on all of $X$. The corresponding gauge group for the parabolic bundle $E$ is

$$
\mathcal{G}=\left\{g \in C^{\infty}(\text { Aut } E) ; \text { respects the flag } \mathcal{F}\right\}
$$

Similarly the gauge group for hermitian bundle $(E, h)$ is defined by

$$
\mathcal{G}_{h}=\left\{g \in \mathcal{G}_{C} ;\left.g\right|_{X-\{p\}} \text { is } h \text {-unitary }\right\} .
$$

The following definitions explain in what sense a metric is adaptive with the parabolic structure.

Definition 3.1. One says that the frame $\left(e_{i}\right)$, which is a basis for the bundle $E$ at $p$, respects the flag structure over $p$ if it is a $C^{\infty}$ local basis in a neighborhood of $p$ for the bundle $E$ and furthermore $E_{p, i}$ is generated by $\left(e_{l-\operatorname{dim}\left(E_{p, i}\right)+1}(p), \ldots, e_{l}(p)\right)$.

Definition 3.2. Suppose $r>1$ and let $\left(\epsilon_{i}\right)$ be a local basis of $C^{\infty}$ sections in a neighborhood of the point $p$. One says that the basis $\left(\epsilon_{i}\right)$ is 
(1) adaptive with the parabolic structure of $E$ if

$$
\epsilon_{i}=g\left(\frac{f_{i}}{|z|^{\alpha_{i}}}\right)
$$

for $g \in D_{2}^{r}$ and $\left(f_{i}\right)$ is a basis for $E$ at $p$ respecting the flag;

(2) adaptive with $(E, h)$ if it is adaptive with $E$ and furthermore it is $h$-orthonormal.

Definition 3.3. The hermitian metric $h$ on $\left.E\right|_{X-\{P\}}$ is adaptive if $(E, h)$ admits an adaptive basis according to the previous definition.

Remark 3.4. By definition it is clear that the bundle $E$ always posses an adaptive basis and the same statement is valid for hermitian bundle $(E, h)$.

Now we bring an example which is illuminating for the above definitions and we will refer to the computation in this example in the next section.

Example 3.5 (construction of an adaptive metric). Let $z$ be a local coordinate for $X$ around $p$ and let $\left(e_{i}\right)_{1 \leq i \leq l}$ be a local basis of sections for $E$ respecting the flag. One can locally define a metric for $E$ as follows:

$$
h=\left(\begin{array}{ccc}
|z|^{2 \alpha_{1}} & & O \\
& \ddots & \\
O & & |z|^{2 \alpha_{l}}
\end{array}\right) .
$$

In fact this metric can be extended smoothly on $X-\{p\}$ and it is clear that the basis $\left(\frac{e_{i}}{|z|^{\alpha_{i}}}\right)$ is adaptive for $(E, h)$.

Furthermore, we assume that $E$ is a holomorphic bundle having $E$ as its underlying $C^{\infty}$ fiber bundle and also we assume that the sections $\left(e_{i}\right)$ are holomorphic sections of $E$.

The associated Chern connection of the metric $h$ can be locally written as

$$
d^{h}=d+h^{-1} \partial h=d+\alpha \frac{\mathrm{d} z}{z},
$$

where $\alpha$ is the diagonal matrix with coefficients $\left(\alpha_{1}, \alpha_{2}, \ldots, \alpha_{l}\right)$. If we use the orthonormal frame $\epsilon_{i}=\frac{f_{i}}{|z|^{\alpha_{i}}}$ instead of $\left(e_{i}\right)$ after some ordinary calculation, we obtain the formula

$$
\bar{\partial}^{E}=\bar{\partial}-\frac{1}{2} \alpha \frac{\mathrm{d} \bar{z}}{\bar{z}}, \quad d^{h}=d+i \alpha \mathrm{d} \theta .
$$

From the above computation it is clear that the curvature of the connection $d^{h}$ vanishes. We say that the holomorphic parabolic bundle $\mathcal{E}$ is decomposable if it admits a holomorphic decomposition $\mathcal{E}=\mathcal{F} \oplus \mathcal{G}$ such that $\mathcal{F}$ and $\mathcal{G}$ are holomorphic sub-bundles and they are equipped with the parabolic structure induced from the parabolic bundle $E$ and furthermore the union of weights of the induced parabolic structures on $\mathcal{F}$ and $\mathcal{G}$ is equal to the weights of the parabolic bundle $\mathcal{E}$.

Now we can describe the notion of parabolicity of a holomorphic bundle in terms of geometry of the bundle itself as follows.

By a parabolic structure for a holomorphic bundle $\mathcal{E}$ over a point $p$ we mean a choice of an adaptive metric $h$ degenerate at $p$ and the type of degeneracy at $p$ determines the weights and the flag structure over cusp point $p$. So we can deal with the notion of parabolicity by using the geometry of the space of singular connections of the bundle $E$. 
With this differential geometric viewpoint of parabolicity we bring a theorem which enlightens the relation between the notion of parabolic degree and the geometry of the holomorphic bundle $\mathcal{E}$ which plays the role of Gauss-Bonnet theorem in the context of parabolic bundles (see [4]).

Theorem 3.6 (Gauss-Chern formula). Suppose $h$ is an adaptive metric on the holomorphic bundle $\mathcal{E}$ and consequently $E$ equipped with a parabolic structure; then for every connection $A \in \mathcal{A}^{r}$ we have

$$
-\frac{1}{2 \pi i} \int_{X} \operatorname{tr}\left(F_{A}\right)=\operatorname{pardeg}(\mathcal{E}) .
$$

Proof. Since $A \in \mathcal{A}^{p}$, in terms of the adaptive basis $\left(\epsilon_{i}\right)$ one has the local expression

$$
A=D+a, \quad a \in D_{1}^{p} .
$$

Let $A_{0}$ be a connection on $E$ smooth on all of $X$; then by a similar computation as in the example above one obtains

$$
A_{0}=d-\alpha \frac{\mathrm{d} r}{r}+b, \quad b \in D_{1}^{p} .
$$

The differential 1 - form $c=A-A_{0}$ which is defined on $X-\{p\}$ and has values in $\operatorname{End}(E)$ has the local expression

$$
c=i \alpha \mathrm{d} \theta+\alpha \frac{\mathrm{d} r}{r}+a-b .
$$

It is easy to see that $\operatorname{tr}\left(F_{A}\right)-\operatorname{tr}\left(F_{0}\right)=d \operatorname{tr}(c)$. Let $B_{\varepsilon}$ be a ball of radius $\varepsilon$ around $p$ with boundary $C_{\varepsilon}$ by integration over $X-B_{\varepsilon}$ and using Stokes theorem we obtain

$$
-\frac{1}{2 \pi i} \int_{X-B_{\varepsilon}}\left(\operatorname{tr}\left(F_{A}\right)-\operatorname{tr}\left(F_{0}\right)\right)=\frac{1}{2 \pi i} \int_{C_{\varepsilon}}(\operatorname{tr}(i \alpha \mathrm{d} \theta)+\operatorname{tr}(a-b)) .
$$

Since $a-b \in L_{1}^{p}$, we have

$$
\int_{C_{\varepsilon}} \operatorname{tr}(a-b) \longrightarrow 0
$$

when $\varepsilon \rightarrow 0$ hence by tending $\varepsilon$ to zero we obtain

$$
-\frac{1}{2 \pi i} \int_{X} \operatorname{tr}\left(F_{A}\right)=\operatorname{tr}(\alpha)-\frac{1}{2 \pi i} \int_{X} \operatorname{tr}\left(F_{0}\right) .
$$

The right-hand side of the above formula is exactly the definition of the parabolic degree and finally Gauss-Chern formula was proved.

Using this differential geometric viewpoint of parabolic bundle we are ready to pose the main theorem in [4] which characterize the stable (semi-stable) parabolic bundles in a differential geometric fashion. For differential geometric proof of a similar theorem concerning ordinary stability, we refer to [5].

Theorem 3.7. Let $\mathcal{E}$ be an indecomposable parabolic bundle equipped with an adaptive hermitian metric $h$. The bundle $E$ is parabolic stable if and only if there exists a connection $A \in \mathcal{A}$ satisfying

$$
* F_{A}=-2 \pi i \mu(\mathcal{E}) .
$$

Moreover, this connection is unique up to the action of the gauge group. 


\section{Support of the product of two conjugacy classes}

Let $C_{\alpha}$ and $C_{\beta}$ be two conjugacy classes in the group $\mathrm{SU}(n)$ and suppose the conjugacy class $\left(C_{\gamma}\right)^{-1}$ occurs in the product of conjugacy classes $C_{\alpha}$ and $C_{\beta}$. Equivalently the identity matrix occurs in the product $C_{\alpha} \cdot C_{\beta} \cdot C_{\gamma}$; we are attempting to characterize all such $\gamma$ 's in the Lie algebra of maximal torus. So in this case we have a representation $\rho: \pi_{1}\left(\mathbb{P}^{1} \backslash\{0,1, \infty\}\right)=\prec$ $a, b, c \mid a b c=1 \succ \rightarrow \mathrm{SU}(n)$ such that $\rho(a) \in C_{\alpha}, \rho(b) \in C_{\beta}$ and $\rho(c) \in C_{\gamma}$. Through this section we choose the integer number $N$ so that all the numbers $N \alpha_{i}, N \beta_{i}$ and $N \gamma_{i}$ are integer numbers.

According to the construction in Section 2 we see that corresponding to this representation $\rho$ there is a special extended bundle over $\mathbb{P}^{1}$ which we named $E_{\rho}^{*}$. Furthermore, this bundle is a parabolic bundle with parabolic structure over the cusp points $0,1, \infty$ by the definition posed in Section 2. Now we are attempting to show that the parabolic bundle $E_{\rho}^{*}$ over $\mathbb{P}^{1}$ is semi-stable. To this aim first we construct a special connection on $E_{\rho}^{*}$ as follows.

As we know from the Section 2 the bundle $E_{\rho}$ over $M=\mathbb{P}^{1} \backslash\{0,1, \infty\}$ is trivial, so it admits a flat connection $\Omega$ on $M$. Although this connection is not defined on parabolic points 0,1 , and $\infty$ similar to the extension of the bundle $E_{\rho}$, we can extend the flat connection $\Omega$ to an appropriate singular connection $\widetilde{\Omega}$ on the extended bundle $E_{\rho}^{*}$ with singularities at parabolic points 0,1 , and $\infty$. For this purpose we consider the singular connection $\omega_{\alpha}$ :

$$
\omega_{\alpha}=\left(\begin{array}{ccc}
\alpha_{1} \frac{d z}{z} & & O \\
& \ddots & \\
O & & \alpha_{n} \frac{d z}{z}
\end{array}\right)
$$

in the neighborhood $U_{\alpha}$ around 0 and similarly we define the singular connection $\omega_{\beta}$ in the neighborhood $U_{\beta}$ around 1:

$$
\omega_{\beta}=P\left(\beta \frac{\mathrm{d} z}{z}\right) P^{-1}
$$

and we also consider the singular connection $\omega_{\gamma}$ in the neighborhood $U_{\gamma}$ around the parabolic point $\infty$.

Now we show that the connections $\Omega, \omega_{\alpha}, \omega_{\beta}$, and $\omega_{\gamma}$ satisfy the compatibility conditions and therefore we can define a global singular connection on the bundle $E_{\rho}^{*}$ with singularities at parabolic points 0,1 , and $\infty$.

To check the compatibility conditions we begin to verify the condition for the two singular connections $\omega_{\alpha}$ and $\omega_{\beta}$; in fact we should verify that on $U_{\alpha} \cap U_{\beta}$ we have

$$
\omega_{\beta}=g_{\alpha \beta}^{-1} \omega_{\alpha} g_{\alpha \beta}+g_{\alpha \beta}^{-1} d g_{\alpha \beta} .
$$

In Section 2 we see that $g_{\alpha \beta}$ is the transition function with $g_{\alpha \beta}(z)=z^{\alpha} P z^{-\beta} P^{-1}$ for the diagonal matrices $\alpha$ and $\beta$ :

$$
\alpha=\left(\begin{array}{ccc}
\alpha_{1} & & O \\
& \ddots & \\
O & & \alpha_{n}
\end{array}\right), \quad \beta=\left(\begin{array}{lll}
\beta_{1} & & O \\
& \ddots & \\
O & & \beta_{n}
\end{array}\right) .
$$


For the right-hand side of the above relation, we have

$$
\begin{aligned}
& \left(P z^{\beta} P^{-1} z^{-\alpha}\right)\left(\alpha \frac{\mathrm{d} z}{z}\right)\left(z^{\alpha} P z^{-\beta} P^{-1}\right) \\
& \quad+P z^{\beta} P^{-1} z^{-\alpha}\left(\mathrm{d}\left(z^{\alpha}\right) P z^{-\beta} P^{-1}+z^{\alpha} P \mathrm{~d}\left(z^{-\beta}\right) P^{-1}\right) \\
& \quad=P z^{\beta} P^{-1}\left(\alpha \frac{\mathrm{d} z}{z}\right) P z^{-\beta} P^{-1}-P z^{\beta} P^{-1}\left(\alpha \frac{\mathrm{d} z}{z}\right) P z^{-\beta} P^{-1}+P\left(\beta \frac{\mathrm{d} z}{z}\right) P^{-1}=\omega_{\beta} .
\end{aligned}
$$

So the compatibility condition for two connections $\omega_{\alpha}$ and $\omega_{\beta}$ has been shown for the other two connections $\omega_{\alpha}, \omega_{\gamma}$ and $\omega_{\beta}, \omega_{\gamma}$; the compatibility condition can be similarly verified.

Now we check the compatibility condition for the connection $\Omega$ with each connection $\omega_{\alpha}$, $\omega_{\beta}$, and $\omega_{\gamma}$; for example we show the compatibility condition between the connection $\Omega$ and $\omega_{\alpha}$ (for the other two connections $\omega_{\beta}$ and $\omega_{\gamma}$, the argument of compatibility is exactly the same).

According to a theorem (see [8]) since the bundle $E_{\rho}$ is trivial, it admits a flat structure $\left(g_{a}, U_{a}\right)$ for which the flat connection $\Omega$ on the bundle $E_{\rho}$ has the representation $\omega_{a}=0$. Let $U_{0}$ be an open set containing 0; according to the triviality of the bundle $E_{\rho}$ it is evident that the transition function $g_{0 \alpha}: U_{0} \cap U_{\alpha} \rightarrow \mathrm{GL}(n, \mathbb{C})$ is equal to $g_{0 \alpha}(z)=z^{\alpha}$.

Now we have to prove that the connection $\omega_{0}=0$ on $U_{0}$ and $\omega_{\alpha}$ on $U_{\alpha}$ is compatible on $U_{0} \cap U_{\alpha}$ in fact we should verify

$$
\omega_{\alpha}=g_{0 \alpha}^{-1} \omega_{0} g_{0 \alpha}+g_{0 \alpha}^{-1} \mathrm{~d} g_{0 \alpha}
$$

by substituting $\omega_{0}=0$ and $g_{0 \alpha}(z)=z^{\alpha}$; the validity of the above formula is trivial because we have

$$
\omega_{\alpha}=z^{-\alpha} \mathrm{d}\left(z^{\alpha}\right)=\alpha \frac{\mathrm{d} z}{z},
$$

which is the definition of the singular connection $\omega_{\alpha}$; thus the compatibility of the connections $\Omega$ and $\omega_{\alpha}$ was verified. Therefore, all the connections $\Omega, \omega_{\alpha}, \omega_{\beta}$, and $\omega_{\gamma}$ are compatible over $\mathbb{P}^{1}$, so we can define a global singular connection $\Omega^{*}$ on the parabolic bundle $E_{\rho}^{*}$ with singularities at parabolic points 0,1 , and $\infty$ of the type $\alpha, \beta$, and $\gamma$, respectively.

It can easily be seen that the curvature of the connection $\omega_{\alpha}$ vanishes and also the connection $\Omega$ has zero curvature, so the curvature of the global singular connection $\Omega^{*}$ introduced above vanishes; hence we have the following theorem.

Theorem 4.1. The parabolic degree of the parabolic bundle $E_{\rho}^{*}$ is zero.

Proof. According to Theorem 3.6, we have

$$
-\frac{1}{2 \pi i} \int_{\mathbb{P}^{1}} \operatorname{tr}\left(F_{\Omega^{*}}\right)=\operatorname{pardeg}\left(E_{\rho}^{*}\right)
$$

but we have $F_{\Omega^{*}}=0$ and consequently $\operatorname{pardeg}\left(E_{\rho}^{*}\right)=0$.

Therefore, by the above argument to a triple $(\alpha, \beta, \gamma)$ of eigenvalues (in which $\gamma$ occurs in the product of two fixed conjugacy classes $C_{\alpha}$ and $C_{\beta}$ ) we associate a flat singular connection with singularity at 0,1 , and $\infty$ of residues $\alpha, \beta$, and $\gamma$, respectively.

The singular flat connection described above induces a singular connection $\Omega_{S}^{*}$ on every sub-bundle $S \subset E_{\rho}^{*}$ with appropriate residues $\alpha_{S}, \beta_{S}$, and $\gamma_{S}$ which is not necessarily flat. In the following we want to prove that for all such sub-bundles $S$ we have $\operatorname{pardeg}\left(\omega_{S}\right) \leq 0$; in other words, the bundle $E_{\rho}^{*}$ is semi-stable. 
Theorem 4.2. The parabolic bundle $E_{\rho}^{*}$ is semi-stable.

Proof. By the argument in this section we see that the bundle $E_{\rho}^{*}$ admits a singular connection $A=\Omega^{*}$ with appropriate singularities at cusp points $0,1, \infty$ and moreover by the construction its curvature is identically zero. Let $S \subset E$ be a holomorphic sub-bundle of the parabolic bundle $E=E_{\rho}^{*}$. So we have the exact sequence of holomorphic bundles

$$
0 \longrightarrow F \longrightarrow E \longrightarrow Q \longrightarrow 0
$$

where $Q$ is the quotient bundle. Using a well-known theorem in differential geometry for example we refer to [8]; the unitary connection $A$ on $E$ described above has the following shape:

$$
A=\left[\begin{array}{c|c}
A_{S} & \beta \\
\hline-\beta^{*} & A_{Q}
\end{array}\right] .
$$

With $A_{F}$ and $A_{Q}$ connections on $F$ and $Q$ and $\beta$ in $\Omega_{2}^{0,1}$. For the corresponding curvature matrix we have

$$
F(A)=\left[\begin{array}{cc}
F\left(A_{S}\right)-\beta \wedge \beta^{*} & \mathrm{~d} \beta \\
-\mathrm{d} \beta^{*} & F\left(A_{Q}\right)-\beta^{*} \wedge \beta
\end{array}\right],
$$

where d : $\Omega^{1}\left(Q^{*} \otimes S\right) \rightarrow \Omega^{2}\left(Q^{*} \otimes S\right)$ is built from $A_{Q}$ and $A_{S}$ and the quadratic terms have a definite sign. For convenience normalize so that ${ }^{*} \operatorname{tr}\left(\beta^{*} \wedge \beta\right)=2 \pi i\|\beta\|^{2}$.

Furthermore, by the construction of this connection we know that the above curvature matrix, $F(A)$, is identically zero. Hence, for the curvature of the connection $A_{S}$ of the subbundle $S \subset E$ we have

$$
F_{S}=F\left(A_{S}\right)=-\beta^{*} \wedge \beta .
$$

Now by Gauss-Chern formula proved in Section 3 we have

$$
\operatorname{pardeg}(S)=\frac{1}{2 \pi i} \int_{\mathbb{P}^{1}} \operatorname{tr}\left(F_{S}\right)=-\frac{1}{2 \pi i} \int_{\mathbb{P}^{1}} \operatorname{tr}\left(\beta^{*} \wedge \beta\right)=-\int_{\mathbb{P}^{1}}\|\beta\|^{2}<0 .
$$

So we proved $\operatorname{pardeg}(S) \leq 0$ and consequently we have $\mu(S) \leq 0=\mu(E)$. Hence, by the above argument we showed that the bundle $E_{\rho}^{*}$ is semi-stable.

The direct consequence of this theorem is the following corollary.

Corollary 4.3. The support of the product of two conjugacy classes in $\mathrm{SU}(n)$ is contained in the set of inequalities, of the form $\mu(S) \leq 0$ where $S$ goes over the sub-bundles of the parabolic bundle $E_{\rho}^{*}$, concerning the semi-stability of the parabolic bundle $E_{\rho}^{*}$.

Notice that the above inequality $\mu(S) \leq 0$ is equivalent to the inequality pardeg $\leq 0$, so for better understanding of the inequalities of this form we should be able to compute the parabolic degree of the sub-bundle $S \subset E_{\rho}^{*}$ or equivalently we should know the parabolic weights induced from the bundle $E_{\rho}^{*}$ to the sub-bundle $S$.

As we mentioned in the definition of semi-stability in the beginning of this paper the induced weights on the sub-bundle $S$ of rank $r$ is as follows.

We follow our important case, the parabolic bundle $E_{\rho}^{*}$ on $\mathbb{P}^{1}$, in this case we know that $\alpha$, $\beta$, and $\gamma$ are the parabolic weights on the cusp points 0,1 , and $\infty$, respectively. For example 
we explain the induced weights on the cusp points 0 for the sub-bundle $S$ and for the other cusp points the argument is exactly the same. To do this let us assume that the flag structure on the fiber over the point 0 in the bundle $E_{\rho}^{*}$ (recall the definition of parabolicity) is

$$
E_{0}=F_{1} E_{0} \supseteqq F_{2} E_{0} \supseteqq \cdots \supseteqq F_{n} E_{0} .
$$

An ascending flag in the fiber $S_{0}$ at marked (cusp) point 0 for the sub-bundle $S$ is obtained by removing from

$$
S_{0} \cap E_{0,1} \subseteq S_{0} \cap E_{0,2} \subseteq \cdots \subseteq S_{0} \cap E_{0, n}=S_{0}
$$

those terms for which the inclusion is not strict (note that since the vector space $S_{0}$ is an $r$ dimensional vector space, exactly $r$ inclusions of the above sequence of inclusions are strict). The parabolic weights for $S$ over 0 are numbers $\alpha_{k_{j}}$, where $k_{j}$ is the minimal index such that $S_{p, j} \subseteq E_{p, k_{j}}$ where $S_{0, j}=S_{0} \cap E_{0, j}$.

So by this explanation the parabolic degree of the sub-bundle $S$ is equal to

$$
\operatorname{pardeg}(S)=\operatorname{deg}(S)+\sum_{i \in I_{S}} \alpha_{i}+\sum_{j \in J_{S}} \beta_{j}+\sum_{k \in K_{S}} \gamma_{k},
$$

where

$$
I_{S}=\left\{i \mid \alpha_{i} \text { is a weight of the sub-bundle } S\right\} .
$$

$J_{S}$ and $K_{S}$ are defined similarly.

Now we are ready to bring our result about the product of two conjugacy classes in terms of an appropriate set of linear inequalities as a corollary of this section.

Corollary 4.4. The support of the product of two conjugacy classes $\alpha$ and $\beta$ is the set of all eigenvalues $\gamma$ which is necessarily contained in the set of linear inequalities of the form

$$
\operatorname{deg}(S)+\sum_{i \in I_{S}} \alpha_{i}+\sum_{j \in J_{S}} \beta_{j}+\sum_{k \in K_{S}} \gamma_{k} \leq 0
$$

where $S$ goes over the sub-bundles of the parabolic bundle $E_{\rho}^{*}$ and the subsets $I_{S}, J_{S}, K_{S}$ are the corresponding indices of the induced parabolic weights for the sub-bundle $S$ as explained above.

To see that this set of linear inequalities are also sufficient to characterize the product of two conjugacy classes we can make use of the convexity theorem for Hamiltonian action of loop groups proved by Meinrenken and Woodward [10], which says that this support, as a subset of maximal torus, is a convex polytope of maximal dimension. Notice that it can be easily verified that all boundary hyperplanes defined by the above linear inequalities are in the support and so by the above-mentioned convexity result we can deduce that the whole convex set defined by the above (IJK)-inequalities are the exact support of the product of two conjugacy classes $C_{\alpha}$ and $C_{\beta}$. Note that some of the above inequalities may be redundant; to see how one can choose an independent set of linear inequalities to describe the support, we refer to $[2,3]$.

We can also prove the sufficiency of the (semi-stability) inequalities by using the correspondence between the moduli of singular flat connections (up to the gauge group action) and the product of conjugacy classes $C_{\alpha}$ and $C_{\beta}$, which we bring in the following. 
Theorem 4.5. Assume that a holomorphic vector bundle $E$ over $\mathbb{P}^{1}$ admits a singular flat unitary connection (with respect to the degenerate metric) with singularity at 0,1 , and $\infty$ whose residues are $\alpha, \beta$, and $\gamma$, respectively. Then $\gamma$ occurs in the product of two conjugacy classes $C_{\alpha}$ and $C_{\beta}$.

Proof. According to Theorem 4.2 this bundle is automatically semi-stable. The monodoromy of the flat connection $\left.\omega\right|_{M}$ gives us a representation of the fundamental group of $M$, namely, $\rho: \pi_{1}(M)=\prec \gamma_{1}, \gamma_{2}, \gamma_{3} \mid \gamma_{1} \gamma_{2} \gamma_{3}=1 \succ \rightarrow \mathrm{SU}(n)$. One can easily show that the condition of the residue around 0 to be $\alpha$ is equivalent to the condition $\rho\left(\gamma_{1}\right) \in C_{\alpha}$ (because locally the connection around 0 has the form $d+i \alpha d \theta)$ and similar conditions are satisfied for singularities 1 and $\infty$. So we have a representation $\rho$ in which $\rho\left(\gamma_{1}\right) \in C_{\alpha}, \rho\left(\gamma_{2}\right) \in C_{\beta}$, and $\rho\left(\gamma_{3}\right) \in C_{\gamma}$ and consequently $\gamma$ occurs in the product of two conjugacy classes $C_{\alpha}$ and $C_{\beta}$.

\section{References}

[1] S. Agnihotri and C. T. Woodward. Eigenvalues of products of unitary matrices and quantum Schubert calculus. Math. Res. Lett., 5 (1998), 817-836.

[2] P. Belkale. Local systems on $\mathbb{P}^{1}-S$ for $S$ a finite set. Composito Math., 129 (2001), 67-86.

[3] P. Belkale. Irredundancy in eigenvalue problems. Preprint arXiv:math/0308026v1, 2003.

[4] O. Biquard. Fibrés paraboliques stables et connexions singulières plates. Bull. Soc. Math. France, 119 (1991), 231-257.

[5] S. K. Donaldson. A new proof of a theorem of Narasimhan and Seshadri. J. Differential Geom., 18 (1983), 269-277.

[6] W. Fulton. Eigenvalues, invariant factors, highest weights and Schubert calculus. Bull. Amer. Math. Soc. (N.S.), 37 (2000), 209-249.

[7] A. A. Klyachko. Stable bundles, representation theory and Hermitian operators. Selecta Math. (N.S.), 4 (1998), 419-445.

[8] S. Kobayashi. Differential geometry of complex vector bundles. Princeton University Press, Princeton, NJ, 1987.

[9] V. Mehta and C. S. Seshdri. Moduli of vector bundles on curves with parabolic structures. Math. Ann., 248 (1980), 205-239.

[10] E. Meinrenken and C. Woodward. Hamiltonian loop group actions and Verlinde factorization. J. Differential Geometry, 50 (1998), 417-469.

Received September 24, 2009

Revised April 14, 2010 